Es bleiben Integrale der unten definierten Art $W_{\tau}^{v}(m, n ; \alpha)$ übrig. Analog kann man bei den Ionenintegralen nach $\varphi_{1}, \varphi_{2}$ und $v_{1}$ (bzw. $v_{2}$ ) integrieren. Außer $W_{\tau}^{v}(m, n ; \alpha)$ bleiben Integrale der Form $G_{\tau}^{v}(m ; \alpha)$ zurück. Diese beiden Integraltypen:

$$
\begin{gathered}
W_{\tau}^{v}(m, n ; \alpha)=\int_{1}^{\infty} \int_{1}^{\infty} Q_{\tau}^{v}\left(\lambda_{+}\right) P_{\tau}^{v}\left(\lambda_{-}\right) e^{-\alpha\left(\lambda_{1}+\lambda_{2}\right)} \\
\cdot \lambda_{1}{ }^{m}{\lambda_{2}}^{n}\left(\lambda_{1}{ }^{2}-1\right)^{v / 2}\left(\lambda_{2}{ }^{2}-1\right)^{v / 2} d \lambda_{1} d \lambda_{2}, \\
G_{l}^{v}(m ; \alpha)=\int_{-1}^{+1} e^{-\alpha \mu} P_{\tau}^{v}(\mu) \mu^{m}\left(1-\mu^{2}\right)^{v / 2} d \mu
\end{gathered}
$$

mit $\lambda=\operatorname{Co} j u ; \mu=\cos v$;

worin $\alpha=\frac{Z}{2} \frac{R}{a_{0}}$ ein Parameter ist, lassen sich nur numerisch auswerten. Man findet sie für $\alpha=1$ bis $\alpha=7$ in ${ }^{10}$.

Da es numerisch einfacher ist, die Integrale $(4 \mathrm{a})$, (4b) und (4c) mit Hilfe der tabellierten Integrale:

$$
\begin{aligned}
& A_{n}(\alpha)=\int_{1}^{\infty} e^{-\alpha \hat{\lambda}} \lambda^{n} d \lambda \\
& \text { mit } \alpha=\frac{Z}{2} \frac{R}{a_{0}}
\end{aligned}
$$

zu berechnen, werden sie auf diese zurückgeführt.

Integral (4a) ist dimensionslos, (4b) bis (4f) haben diè Dimension $\frac{e^{2}}{a_{0}} Z$, worin $Z$ die wählbare Kernladungszahl in den Eigenfunktionen $\left(Z=2 a_{0} \delta\right)$ ist.

Gemäß unserer Definition von $\vartheta_{\mathrm{a}}$ und $\vartheta_{\mathrm{b}}$ bestehen folgende Identitäten innerhalb der einzelnen Integraltypen (auf die bereits oben hingewiesen wurde):

$$
\begin{aligned}
& S_{\alpha \beta}=S_{\beta \alpha} ; \\
& K_{\alpha \beta}=K_{\beta \alpha} ; \\
& \text { aber: } \quad J_{\alpha \beta} \neq J_{\beta \alpha} ; \\
& C_{\alpha \beta \gamma \delta}=C_{\gamma \beta \alpha \delta}=C_{\alpha \delta \gamma \beta}=C_{\gamma \delta \alpha \beta} \\
& \quad=C_{\delta \gamma \beta \alpha}=C_{\delta \alpha \beta \gamma}=C_{\beta \gamma \delta \alpha}=C_{\beta \alpha \delta \gamma} ; \\
& A_{\alpha \beta \gamma \delta}=A_{\beta \alpha \delta \gamma}=A_{\gamma \delta \alpha \beta}=A_{\delta \gamma \beta \alpha} ; \\
& L_{\alpha \beta \gamma \delta}=L_{\gamma \beta \alpha \delta} .
\end{aligned}
$$

Die vollständigen Formeln für die Integrale sind in Tab. 1 zusammengestellt. Tab. 2 enthält die numerischen Werte.

Der Verf. möchte Hrn. Prof. Dr. K. Wirtz für die Anregung zu dieser Arbeit und die zahlreichen wertvollen Diskussionen und Hinweise herzlichst danken. Besonderer Dank gebührt auch Frl. Dr. E. T r e f $\mathrm{ftz}$, die zu sämtlichen Integrationen Kontrollrechnungen durchführte, und der Rechengruppe der astro-physikalischen Abteilung des Max-Planck-Institutes für Physik für die Hilfe bei den zeitraubenden numerischen Auswertungen.

\title{
Quantenelektrodynamische Selbstenergie und exakte Lösungen der Schrödinger-Gleichung I*
}

\author{
Von H. SALECKER ${ }^{* *}$ \\ (Z. Naturforschg. 5 a, 431-438 [1950]; eingegangen am 7. August 1950)
}

\begin{abstract}
Im ersten Abschnitt wird über ein Verfahren zur Behandlung der Entartung in der Quantentheorie der Wellenfelder und insbesondere über den Einfluß der Entartung auf die Selbstenergie des Elektrons berichtet. Es ergibt sich dabei, daß die Behandlung der Entartung keine Änderung der früher erhaltenen Ergebnisse bedingt. Im zweiten und dritten Abschnitt wird die Herkunft der Selbstenergiedivergenz untersucht. Nach einer störungstheoretischen Behandlung im Abschnitt 2 wird mit Hilfe einiger einfacher Beispiele gezeigt, daß es keineswegs selbstverständlich ist, aus der Divergenz der zweiten Näherung auf die Divergenz des exakten Selbstenergieausdrucks zu schließen. Anschließend wird die Frage nach dem Ursprung der Divergenzschwierigkeiten in der Quantenelektrodynamik mit Hilfe einer exakten Methode dahingehend entschieden, daß diese aus den Grundlagen der Theorie stammen; d. h. die zugehörige Schrödinger-Gleichung besitzt in Strenge gar keine Lösung zu endlichen Energieeigenwerten. Diese Ergebnisse sind auch für die neue kovariante Formulierung von Tomonaga und $\mathrm{S} \mathrm{ch}$ w in $\mathrm{g}$ e $\mathrm{r}$ gültig.
\end{abstract}

Schon oft ist auf die Schwierigkeiten hingewiesen $\mathrm{S}_{\text {worden, die der Behandlung quantisierter Feld- }}$ theorien beim Vorhandensein von Wechselwirkungs-

* Gekürzte Diss. Tübingen 1949. Teil I und II. gliedern entgegentreten. Wegen der großen Kompliziertheit der zugehörigen $\mathrm{S} \mathrm{chröding} \mathrm{er-Glei-}$

** Jetzt am Institut für theoretische und angewandte Physik der Technischen Hochschule Stuttgart. 
chung ist es bisher nur möglich gewesen, diese mit Hilfe der Störungstheorie zu lösen. Die dabei sich ergebenden Entwicklungen für Selbstenergien und Wirkungsquerschnitte divergieren in allen Gliedern bis auf die jeweils niedrigstmögliche Näherung. Die letztere jedoch liefert z. B. in der Quantenelektrodynamik Ergebnisse, die mit dem Experiment innerhalb der Fehlergrenzen übereinstimmen. Die Gründe für diesen merkwürdigen Sachverhalt können zunächst in zwei ganz verschiedenen Richtungen gesucht werden. Einmal kann die Benützung der Störungstheorie und der damit verbundenen Potenzreihenentwicklung nach dem Wechselwirkungsparameter die Ursache sein, zweitens aber können die auftretenden Divergenzen auch aus den Grundlagen der Theorie stammen, so daß eine exakte Lösung gar nicht vorhanden ist. Während die letzte Möglichkeit einer Reihe von früheren Versuchen zur Abänderung der Theorie zugrunde gelegt war, sind besonders in neuerer Zeit einige formale Versuche zur Beseitigung der Schwierigkeiten unternommen worden, die die erste Möglichkeit ernstlich in Betracht ziehen. Hier sind besonders Arbeiten von H. W. P e n g ${ }^{1,2}$ und Stellen einer neueren Arbeit von $\mathrm{Dir} \mathrm{a} \mathrm{c}^{3} \mathrm{zu}$ nennen, ohne daß diese Frage bisher endgültig in einem bestimmten Sinne entschieden wurde.

In der vorliegenden Arbeit und einer folgenden ${ }^{4}$ soll nun eine Klärung dieses Fragenkomplexes versucht werden. Der erste Abschnitt berichtet über ein an anderer Stelle ${ }^{5}$ ausführlich wiedergegebenes Verfahren zur Behandlung der Entartung in der Quantentheorie der Wellenfelder und insbesondere über den Einfluß der Entartung auf die Selbstenergie des freien Elektrons, wobei immer noch die Anwendbarkeit der formalen Störungsrechnung vorausgesetzt wird. Dabei ergibt sich, daß die Entartung auf die sogenannte transversale Selbstenergie, die durch die Wechselwirkung des Elektrons mit den Lichtquanten verursacht wird, in zweiter Näherung keinen Einfluß hat. Die grundsätzlichen Schwierigkeiten werden also durch die Entartung nicht beseitigt oder verringert.

Im zweiten und dritten Abschnitt wird die Herkunft der Selbstenergiedivergenz untersucht. Dazu ist es zunächst nötig, einiges vom bereits Bekannten

1 H. W. P e n g, Nature [London] 154, 544 [1944].

2 H. W. P e n g, Proc. Roy. Soc. [London], Ser. A 186, 119 [1946].

3 s. die erste unter 17 genannte Arbeit, S. 12-13.

4 H. S a le c k e r, Z. Naturforschg. 5 a, Heft 9 [1950], im folgenden als II zitiert. von neuem zu betrachten. Mit dessen Hilfe wird dann der Ursprung der zusätzlichen quantenmechanischen Selbstenergie festgestellt. Hierbei ist, wie öfters in der vorliegenden Arbeit, bei mehr ins einzelne gehenden Rechnungen die $\mathrm{H}$ e is e n b e r g - P a u li sche Formulierung ${ }^{6}$ der Quantenelektrodynamik ohne Auffüllung der negativen Energieniveaus also ohne Löchertheorie zugrunde gelegt, da für die nähere Analyse der charakteristischen Schwierigkeiten quantisierter Feldtheorien die Korrespondenz mit der klassischen Theorie wichtig ist, die der Positrontheorie nicht unmittelbar zur Verfügung steht. Zwar werden durch den Übergang zur Positrontheorie die quantitativen Verhältnisse im einzelnen stark verändert, doch sind die charakteristischen Schwierigkeiten überall ganz entsprechend, so daß unsere Überlegungen (insbesondere die allgemeinen in II) nicht auf die spezielle Form der Theorie beschränkt bleiben. Auf hierbei eintretende Unterschiede wird noch jeweils hingewiesen. Mit Hilfe einiger einfacher Beispiele wird weiterhin gezeigt, daß es keineswegs selbstverständlich ist, aus der Divergenz der zweiten Näherung auch auf die Divergenz des exakten Selbstenergieausdrucks zu schließen, wie es verschiedentlich gemacht wurde. Anschließend wird die Frage nach dem Ursprung der Divergenzschwierigkeiten untersucht und dahingehend entschieden, daß diese den Grundlagen der Theorie enistammen, d. h. daß die zugehörige Schrödinger-Gleichung in Strenge gar keine Lösung zu endlichen Energieeigenwerten besitzt.

\section{Der Einfluß der Entartung}

Es ist kürzlich von H. W. P e n g ${ }^{1,2}$ darauf hingewiesen worden, daß die ungestörten Zustände in allen quantisierten Feldtheorien entartet sind, d. h. daß zu jedem Energieeigenwert viele verschiedene Eigenfunktionen gehören und daß deshalb das Störungsverfahren geändert werden müßte. Weiterhin wird der folgende Satz angeführt ${ }^{7}$ : „Wenn einige Zustände des ungestörten Systems entartet sind (genau oder fast entartet), dann können die Eigenfunktionen $\psi_{a}$ des gestörten Systems nicht mit Rücksicht nach der Störung in eine Potenzreihe von der Form

$$
\psi_{a}=\phi_{a}^{(0)}+\psi_{a}^{1}+\psi_{a}^{2}+\ldots
$$

${ }^{5}$ H. S a l e c k e r, Diss. Tübingen 1949, Teil I.

6 W. H e is e n berg u. W. Pa u li, Z. Physik 56, 1 [1929].

7 s. Fußnote 2, S. 126. 
entwickelt werden, wo $\psi^{1}{ }_{a}$ die erste Potenz der Wechselwirkungskonstanten als Faktor enthält, $\psi^{2}{ }_{a}$ die zweite Potenz usw. “ Außerdem wird behauptet, daß bei konsequenter Durchführung der Störungstheorie, d. h. Behandlung der Entartung, die bekannten Divergenzschwierigkeiten beseitigt würden. Nun ist der Hinweis auf die Entartung bei quantisierten Feldtheorien durchaus notwendig, denn in der bisherigen Lösung der zugehörigen Schrödinger-Gleichung mit Hilfe der Störungstheorie wurde der Entartung keineswegs Rechnung getragen, im Gegensatz zur ursprünglichen Schrödingerschen Störungstheorie. Jedoch der oben angeführte Satz von $\mathrm{Peng}$ ist in dieser Form sicher nicht ganz einwandfrei, denn auch in der Schrödingerschen Störungstheorie entarteter Systeme wird eine Potenzreihenentwicklung der gestörten Eigenfunktion nach dem Wechselwirkungsparameter erhalten. Allerdings muß die Ausgangseigenfunktion des ungestörten Systems erst durch das Säkularproblem bestimmt werden, und wenn die Entartung nicht nach einem Schritt aufgehoben ist, sind auch die weiteren Verbesserungen durch Säkularprobleme eindeutig festzulegen. Dieses Vorgehen der Schrödingerschen Störungsrechnung kann leicht in die Quantentheorie der Wellenfelder übertragen und insbesondere auf die Berechnung der sogenannten transversalen Selbstenergie des Elektrons zweiter Näherung angewendet werden ${ }^{5}$. Doch sollen an dieser Stelle die diesbezüglichen Entwicklungen nicht im einzelnen dargestellt, sondern nur über den Gang der Rechnung und die Ergebnisse berichtet werden. Dabei wird außer den durch die Behandlung der Entartung bedingten Erweiterungen des gewöhnlichen Verfahrens keine weitere Änderung vorgenommen, um den Einfluß der Entartung klar herauszustellen. Zunächst wird ein für die Quantenelektrodynamik verallgemeinerter Greenscher Satz aufgestellt, mit dessen Hilfe sich ganz analog wie bei der Schrödingerschen Störungstheorie für stationäre Zustände eine Lösbarkeitsbedingung für diejenige aus der allgemeinen Schrödinger-Gleichung abgeleitete Gleichung ergibt, die die erste Näherung der Wellenfunktion bestimmt. Diese Gleichung ist bekanntlich eine inhomogene Gleichung, in der auch die erste Näherung des Energieeigenwertes auftritt. Die Lösbarkeitsbedingung besagt dabei, daß die rechte Seite der inhomogenen Gleichung orthogonal sein muß zu sämtlichen Lösungen der homogenen (ungestörten) Gleichung. Beschränkt man sich jetzt auf solche Anfangszustände des ungestörten Systems, bei denen keine Lichtquanten vorhanden sind, so ist der
Energieeigenwert $E_{0}$ gleich der Energie des DiracElektrons, und zu jedem Wert von $E_{0}$ gibt es viele verschiedene Eigenfunktionen, die den verschiedenen Richtungen des Impulses des Elektrons und den beiden Spinzuständen entsprechen. Das ungestörte Problem ist also hoch entartet. Entsprechend dem Falle der Entartung in der Schrödingerschen Störungstheorie muß nun die passende Ausgangseigenfunktion des ungestörten Zustandes erst durch das sogenannte Säkularproblem bestimmt werden. Dazu dient die Lösbarkeitsbedingung. Als Eigenfunktion zum ungestörten Zustand $E_{0}$ wird eine Linearkombination aller entarteten Eigenfunktionen eingesetzt. Läßt man das übliche Periodizitätsvolumen $V$, in das man sich das System in der Quantentheorie der Wellenfelder meist eingeschlossen denkt, über alle Grenzen wachsen, so daß man auch kontinuierliche Eigenwertspektren erhält, so liefert die Lösbarkeitsbedingung jetzt eine homogene Fredholmsche Integralgleichung 2. Art (Eigenwertproblem) mit den Koeffizienten der entarteten Eigenfunktionen als Unbekannten und der ersten Näherung des gesuchten Energieeigenwerts $E_{1}$ als Eigenwert. Im vorliegenden speziellen Fall der Selbstenergieberechnung verschwindet jedoch die linke Seite, so daß $E_{1}=0$ folgt, wie zu erwarten ist. Zur Selbstenergie können ja nur zweistufige und höhere Prozesse gerader Ordnung beitragen. Die Berechnung der ersten Näherung der Wellenfunktion geschieht nun ganz in der üblichen Weise durch Entwicklung nach dem vollständigen Orthogonalsystem der ungestörten Eigenfunktionen. Bei der Bestimmung der zweiten Näherung ergibt sich wieder dieselbe Lösbarkeitsbedingung unter Ersetzung der ungestörten Wellenfunktion durch die erste Näherung der gestörten, da die Entartung nicht verringert ist. Damit erhält man wieder eine Integralgleichung von dem eben erwähnten Typ mit der zweiten Näherung des Energieeigenwerts als Eigenwert. In dem vorliegenden speziellen Fall der Selbstenergieberechnung reduziert sie sich jedoch auf eine einfache Gleichung, die den schon von $W$ a 11 e r ${ }^{8}$ bzw. Weißkopf ${ }^{9}$ gefundenen Ausdruck für die Selbstenergie liefert.

Es ergibt sich somit also, daß die Entartung auch in zweiter Näherung weder aufgehoben noch verringert ist und daß die Behandlung der Entartung keinen Einfluß hat auf die divergierende transversale Selbstenergie des Elektrons zweiter Näherung. Auch rein anschaulich ist das eben erhaltene Resultat durchaus zu erwarten, da bei ganzer oder teilweiser Auf-

8 J. W a ll e r, Z. Physik 62, 673 [1930].

9 V. F. W e iß k o p f, Physic. Rev. (2) 56, 72 [1939]. 
hebung der Entartung die Energie des gestörten Systems auch von der Richtung des Impulses des Elektrons abhängen würde, was keinen physikalischen Sinn hätte. Unsere Feststellung berührt sich hier mit einer neueren Arbeit von J. $\mathrm{H}$ a $\mathrm{m}$ il t o $\mathrm{n}^{10}$, der ebenfalls der Entartung keine bedeutende Rolle zuschreibt.

Bei dieser Feststellung erhebt sich sogleich die Frage nach dem Verhalten der höheren Näherungen. Doch ist die Beantwortung der letzteren nicht von großer physikalischer Bedeutung. Die unveränderte Divergenz der ersten Näherung bedeutet nämlich entweder, daß auch der exakte Selbstenergieausdruck divergiert, d. h. daß die zugehörige Schrödinger-Gleichung in Strenge gar keine Lösung besitzt, oder daß mindestens die benutzte Methode, also die Störungstheorie, auch bei Berücksichtigung der Entartung unpassend ist. Zwischen diesen beiden Möglichkeiten kann man aber durch eine Untersuchung der höheren Näherungen nicht entscheiden. Die Klärung dieser fundamentalen Frage, der wir uns im folgenden zuwenden wollen, muß also in anderer Weise erfolgen.

\section{Die transversale Selbstenergie zweiter Näherung}

Wie im Abschnitt 1 berichtet wurde, liefert auch eine die Entartung berücksichtigende konsequente Störungstheorie in zweiter Näherung dieselbe divergierende transversale Selbstenergie, die im Falle der Quantenelektrodynamik ohne Auffüllung der negativen Energieniveaus, die wir wegen der ihr unmittelbarer als der Positrontheorie zur Verfügung stehenden Korrespondenz mit der klassischen Theorie zunächst betrachten wollen, schon von $\mathrm{W}$ a ll e $\mathrm{r}^{8}$ berechnet wurde und die für das makroskopisch ruhende Elektron lautet

$$
E_{2}{ }^{0}=\frac{e^{2}}{\hbar c} \frac{1}{\pi \mu} \int_{0}^{\infty} k d k .
$$

Hier bedeuten $E_{0}$ die Energie des ungestörten Elektrons, $k=\hbar v$ die Lichtquantenenergie, $\mu=m c^{2}$ die Ruhenergie des Dirac-Elektrons, $e$ die Elementarladung, $\hbar=h / 2 \pi$ ( $h$ Plancksches Wirkungsquantum) und $c$ die Lichtgeschwindigkeit. Der Ausdruck (1.1) divergiert quadratisch. Im Falle des makroskopisch bewegten Elektrons tritt noch eine vom Bewegungszustand abhängige lineare Divergenz dazu. Geht man mit $\hbar \rightarrow 0$ zur klassischen Theorie über und beachtet $k=\hbar v$, so verschwindet die quadratische Divergenz (1.1), während die vom Bewegungszustand abhängige

10 J. Hảmilto n, Proc. physic. Soc. 59, 917 [1947]. lineare Divergenz unverändert bleibt. Zusammen folgt für das ruhende klassische Elektron $E_{2}=0$, d. h. die transversale Selbstenergie verschwindet dann, wie es sein muß. Man erhält also, daß die quadratische Divergenz (1.1) quantenmechanischen Ursprungs ist, während die lineare Divergenz schon in der klassischen Theorie vorkommt. Nun ergeben sich für ein punktförmiges bewegtes Elektron auch bereits klassisch durch Lorentz-Transformation aus der elektrostatischen Selbstenergie zusätzliche lineare Divergenzen, die für das ruhende Elektron verschwinden und die die Energie darstellen, die durch das magnetische Feld des bewegten Elektrons hervorgerufen wird. Die Quantelung besteht jetzt beim Dirac-Elektron darin, daß man die Wurzel in der klassischen HamiltonFunktion in der von Dirac angegebenen Weise auszieht, die Geschwindigkeit $\mathfrak{v}$ durch den Diracschen Matrixvektor $c \vec{\alpha}$ sowie $\sqrt{1-v^{2} / c^{2}}$ durch die Matrix $\beta$ ersetzt und die bekannten Vertauschungsbeziehungen für Ort und Impuls einführt. Wie S c h r ö d i n g e r ${ }^{11}$ gezeigt hat, bedeutet die Ersetzung von $\mathfrak{v} / c$ durch $\vec{\alpha}$, daß sich das Elektron immer mit Lichtgeschwindigkeit bewegt. Dieser Schrödingerschen Zitterbewegung überlagert sich die makroskopische translatorische Bewegung. Das Elektron ist also immer von einem Magnetfeld umgeben wie auch von einem alternierenden elektrischen Feld, den Feldern des sogenannten Elektronenspins. Es ist deshalb zu erwarten, daß hier die klassischen zusätzlichen Divergenzen immer bestehen. Die nähere Analyse der einzelnen Terme, die von Weißkopf ${ }^{9}$ in der zweiten Näherung durchgeführt wurde, zeigt jedoch, daß sich die Beiträge der elektrischen und magnetischen Felder des Spins in zweiter Näherung aufheben, so daß hiervon kein Beitrag zur Selbstenergie resultiert (im Gegensatz zur Theorie des Positrons, wo sich in diesem Falle ein quadratisch und ein logarithmisch divergierender Bestandteil ergeben; die quadratische Divergenz hebt dann insgesamt in der zweiten Näherung die sogleich zu besprechende Divergenz der Feldschwankungen auf, so daß nur eine logarithmische Divergenz übrigbleibt ${ }^{9}$ ).

In der Quantenelektrodynamik kommt jedoch zu der Quantisierung des Elektrons noch die Quantisierung des Feldes. Diese verursacht das Bestehen einer Unschärfebeziehung zwischen der Anzahl der Lichtquanten $N$ und der Feldstärke $E$, da beide Größen nicht kommutieren,

$$
\triangle N \triangle E \sim E .
$$

11 E. S c h rö di ng e r, Berliner Ber. 1930, 418 [1930]. 
Dabei bedeutet $\Delta N$ die Unbestimmtheit in der Kenntnis der Lichtquantenzahl und $\Delta E$ diejenige der Feldstärke. Wenn $N$ einen gegebenen Wert annimmt, d. h. $\Delta N=0$, hat die Feldstärke danach keine bestimmte Größe, sondern schwankt um einen Mittelwert; also auch wenn $N=0$ ist, d. h. im leeren Raum wird die Feldstärke beständig schwanken (Nullpunktsschwankungen, Bohr und Ros enf eld ${ }^{12}$ ). Da die Lichtquantenzahl bei der Selbstenergieberechnung bestimmt ist, ergeben die zugehörigen Feldschwankungen erzwungene Schwingungen des punktförmigen Elektrons mit einer Energie, die in zweiter Näherung quadratisch divergiert (daß dieser Term quadratisch divergiert, wurde schon von $\mathrm{W}$ e i $ß \mathrm{k} \mathrm{op} \mathrm{f}^{9}$ gezeigt). Dieser Beitrag liefert also die transversale Selbstenergie des ruhenden Elektrons, die in (1.1) angegeben ist.

\section{Betrachtung der Selbstenergie ohne Störungstheorie}

Die bisherige Behandlung der Selbstenergie in zweiter Näherung der Störungstheorie läßt die Frage offen, ob die beobachteten Divergenzen aus den Grundlagen der Theorie stammen oder vielleicht ganz oder teilweise nur dadurch verursacht sind, daß die formale Störungsrechnung in der Quantenelektrodynamik nicht anwendbar ist und daß deshalb die mathematischen Methoden gewechselt werden müssen. In der Tat kann man leicht Beispiele für den letzteren Fall angeben. Interessant ist dazu das Folgende, daß außerdem noch verschiedene Züge der Theorie veranschaulicht. Durch eine exakte Berechnung sei etwa für die Selbstenergie eines ruhenden ausgedehnten Elektrons mit dem Radius $a$ gefunden

$$
W=m c^{2} \frac{x}{1+x}=\mu \frac{x}{1+x} .
$$

Wird dabei $x=\left(e^{2} / \hbar c\right)(1 / \pi)(\hbar / a m c)^{2}=\left(e^{2} / \hbar c\right)$ $\left(1 / \pi \mu^{2}\right)(\hbar c / a)^{2}$ gesetzt, so erhält man bei Entwicklung nach Potenzen der Feinstrukturkonstanten $\left(e^{2} / \hbar c\right)$

oder

$$
W=\mu x\left(1-x+x^{2}-x^{3}+-\ldots\right)
$$

$$
W=\frac{e^{2}}{\hbar c} \frac{1}{\pi \mu}\left(\frac{\hbar c}{a}\right)^{2}-+\ldots
$$

d. h. bei Übergang zum punktförmigen Elektron $(a \rightarrow 0)$ divergiert der Ausdruck in erster nicht verschwindender Näherung wie der Ausdruck (1. 1), der die Selbstenergie des ruhenden Elektrons in der

$12 \mathrm{~N}$. B ohr u. L. Ros e nfeld, Math. fysik. Medd. Kopenhagen 12, Nr. 8 [1933].
Quantenelektrodynamik ohne Löchertheorie angibt, während aus dem exakten Ausdruck (1.2) mit $a \rightarrow 0$, d. h. $x \rightarrow \infty$, folgt

$$
W=m c^{2} .
$$

Wir haben also hier ein Beispiel dafür, daß die auftretenden Divergenzen vollkommen durch die (ungerechtfertigte) Anwendung der Entwicklungsmethode verursacht sind, während der exakte Ausdruck auch im Falle des verschwindenden Teilchenradius einen vernünftigen Wert liefert. Setzt man übrigens in dem angegebenen Beispiel

$$
x=\left(e^{2} / \hbar c\right) \ln (\hbar / m c a),
$$

so erhält man für $W$

$$
W=m c^{2} \frac{x}{1+x}=m c^{2} \frac{e^{2}}{\hbar c} \ln \frac{\hbar}{m c a}-\ldots .
$$

Das ist gerade der von We i $\beta \mathrm{k}$ o $\mathrm{pf}^{9}{ }^{9}$ in der Positrontheorie gefundene Ausdruck für die zweite Näherung der Selbstenergie. Bei endlichem Teilchenradius $a$ sind die einzelnen Glieder in der Reihe (1.3) alle endlich, jedoch konvergiert (1.3) nicht für jedes $a$, sondern nur für $|x|<1$, da (1.3) die wohlbekannte geometrische Reihe darstellt. Das liefert die Bedingung für den Teilchenradius $a$

$$
a>\frac{e}{\sqrt{\hbar c}} \frac{1}{\sqrt{\pi}} \frac{\hbar}{m c}
$$

für das Beispiel zur gewöhnlichen Quantenelektrodynamik (ohne Auffüllung der negativen Energieniveaus) und von

$$
a>\frac{\hbar}{m c} e^{-\hbar c / e^{2}}
$$

in dem Beispiel zur Positrontheorie. Es ist interessant zu bemerken, daß für die gewöhnliche Quantenelektrodynamik solche Verhältnisse tatsächlich von Gustafs on ${ }^{\mathbf{1 3}}$ und bei der Positrontheorie sogar genau (1. 7) von W e i $ß \mathrm{k} \mathrm{o} \mathrm{p} \mathrm{f}^{9}$ als Konvergenzbedingung für den Teilchenradius $a$ gefunden wurden. Dabei beruht die erstere Untersuchung auf einer exakten Methode, die letztere dagegen nur auf einer Untersuchung mit Hilfe der Störungstheorie, so daß damit noch nichts zur exakten Lösung in der Positrontheorie gesagt ist.

Das dargelegte Beispiel gibt noch eine Ergänzung $\mathrm{zu}$ einem kürzlich von $\mathrm{R} \mathrm{a} \mathrm{a} \mathrm{h}^{14}$ ausgesprochenen Gedanken. Racah betrachtet die Untersuchung von W e i $ß \mathrm{k} \mathrm{op} \mathrm{f}^{9}$ zur Selbstenergie des Elektrons in der

13 T. G u s t a f s o n, Ark. Mat., Astronom. Fysik Ser. A, 26, Nr. 15 [1939].

14 G. R a c a h, Physic. Rev. (2) 70, 406 [1946]. 
Positrontheorie, wo unter anderem auch festgestellt wird, daß die Divergenz in jeder Näherung logarithmisch ist, wie das mit Hilfe unseres Beispiels in der speziellen Form (1.5) erläutert wurde. Außerdem gibt er Hinweise darauf, daß die Berechnung der höheren Näherungen ebenfalls wie (1.5) eine alternierende Reihe liefert. Aus diesen beiden Gründen hält er es weiterhin für wahrscheinlich, daß die Divergenz in der Löchertheorie nur durch die Entwicklungsmethode verursacht ist und in Wirklichkeit gar nicht besteht. Er schließt jetzt, daß wenn der exakte Ausdruck für die Selbstenergie

lautet und

$$
W=m c^{2} f\left(\frac{\hbar}{m c a}, \frac{e^{2}}{\hbar c}\right)
$$

$$
\lim _{a \rightarrow 0} f\left(\frac{\hbar}{m c a}, \frac{e^{2}}{\hbar c}\right)=g\left(\frac{e^{2}}{\hbar c}\right),
$$

$\mathrm{da} ß$ dann die Idee der rein elektromagnetischen Masse $g\left(e^{2} / \hbar c\right)=1$ erfordert, und daß diese Gleichung den Wert der Feinstrukturkonstanten festlegen wird, wobei der sich dabei ergebende Wert von $e^{2} / \hbar c$ über die Brauchbarkeit oder Unbrauchbarkeit der Idee der rein elektromagnetischen Masse durch seine Übereinstimmung oder seinen Widerspruch mit dem experimentell bekannten entscheidet. Wir sehen nun aus unserem Beispiel (1.3), daß die logarithmische Divergenz keineswegs in besonderer Weise für eine Konvergenz des exakten Ausdrucks (1.2) spricht, daß vielmehr dieser Ausdruck für jede beliebig mit $a \rightarrow 0$ gegen $\infty$ divergierende Größe $x$ konvergiert. Ersetzt man (1.2) durch

$$
W=m c^{2} \frac{x}{1-x}=m c^{2} x\left(1+x+x^{2}+x^{3}+\ldots\right),
$$

so erhält man eine Entwicklung mit nur positiven Vorzeichen, zu der der exakte Ausdruck ebenfalls mit $a \rightarrow 0$ (d. h. $x \rightarrow \infty$ ) konvergiert. Auch ergibt der exakte Ausdruck (1.2) mit $a \rightarrow 0$ vollkommene Unabhängigkeit von der Feinstrukturkonstanten $e^{2} / \hbar c$. Die Idee der rein elektromagnetischen Masse legt also hier den Wert von $e^{2} / h c$ keineswegs fest. Es folgt deshalb, daß auch dann, wenn keiner der von Racah angeführten Gründe vorhanden wäre, ebenfalls Konvergenz des exakten Ausdrucks möglich sein würde, und daß dieser sogar so beschaffen sein könnte, daß die Feinstrukturkonstante bei $a \rightarrow 0$ dort gar nicht vorkommt.

Um nun die Frage der Divergenz oder Konvergenz des exakten Selbstenergieausdrucks in der gewöhnlichen Quantenelektrodynamik zu klären, kann man zunächst die Korrespondenz mit der klassischen Theorie zur Hilfe nehmen, obgleich diese nach den obigen Ausführungen nur über einen Teil der Divergenzen Auskunft geben kann. Hier wird nun die Bewegung eines geladenen Teilchens, das wir uns vorerst mit endlichem Radius $a$ vorstellen wollen, durch die Lorentzsche Bewegungsgleichung bestimmt

$$
\frac{d}{d t}(m \mathfrak{v})=\int \varrho\left(\mathfrak{F}+\frac{1}{c} \mathfrak{y} \times \mathfrak{h}\right) d \tau,
$$

wobei $\varrho$ die Ladungsdichte darstellt. Dabei ist für $\mathfrak{F}$ und $\mathfrak{S}$ das gesamte Feld einzusetzen, d. h. das von anderen Ladungen usw. hervorgerufene äußere Feld und das Feld des betrachteten Teilchens selbst, das z. B. die Reaktionskraft der Strahlung enthält. Wertet man jetzt das Integral auf der rechten Seite aus, so erhält man bekanntlich aus den die Eigenfelder enthaltenden Gliedern unter anderem einen der Beschleunigung proportionalen Term, der die elektromagnetische Masse des Elektrons darstellt und der mit $a \rightarrow 0$ linear divergiert. Die Eigenkräfte in der Lorentzschen Gleichung (1.8) sind also für punktförmige Teilchen unendlich groß, so daß diese Gleichung bereits keine eigentliche Lösung besitzt. Doch kann man sich in diesem Falle noch verhältnismäßig leicht helfen, indem man unter Benutzung von gewissen Kunstgriffen die Bewegungsgleichung in anderer Weise als (1. 8) hinschreibt ${ }^{15}$. Auch ist es für die praktische Rechnung von konkreten Problemen bekanntlich möglich, die Eigenkräfte in (1.8) einfach wegzulassen, da ihr Einfluß in Wirklichkeit im allgemeinen klein ist, wenn man, wie üblich, für die Ruhmasse $m_{0}$ die experimentelle Masse einsetzt, wobei die elektromagnetischen Trägheitsglieder mit berücksichtigt sind. Man kann also für $\mathfrak{\mathfrak { E }}$ und $\mathfrak{\mathfrak { C }}$ in (1.8) das äußere Feld allein einsetzen, um eine gute Näherung zu erhalten.

Bei der daran anschließenden Hamiltonschen Formulierung tritt nach Elimination der longitudinalen Wellen die Schwierigkeit wieder ganz klar zutage in Gestalt des Coulomb-Terms $1 / 2 \sum_{i, j}\left(e_{i} e_{j} / r_{i j}\right)$, der die statischen Selbstpotentiale $1 / 2 \sum_{i}\left(e_{i} e_{i} / r_{i}\right)$ enthält, so daß in der Hamilton-Funktion ein Bestandteil $n \cdot \infty$ ( $n$ Anzahl der Teilchen) vorkommt. Ein einfaches Fortlassen dieses Ausdrucks zerstört bereits den von $\mathrm{He}$ isenberg und $\mathrm{Pauli}^{6}$ bei der Aufstellung der Quantenelektrodynamik geführten Beweis der relativistischen Invarianz ${ }^{16}$. Die Forderung relativisti-

15 P. A. M. D i r a c, Proc. Roy. Soc. [London], Ser. A, 167, 148 [1938].

16 Vgl. hierzu etwa den Artikel von W. Pauli, Handbuch der Physik 24/1. Springer-Verlag, Berlin 1933, S. 261 bis 266 . 
scher Invarianz der Theorie war ja bekanntlich einer der Hauptgründe für die Einführung des Punktelektrons. Doch läßt sich das Streichen der statischen Selbstenergie bzw. ihr Einschluß in die experimentell bestimmte Masse des Elektrons auf anderem Wege in durchaus invarianter Weise rechtfertigen, wie man später gesehen hat. Dies leistet etwa der WentzelDiracsche $\lambda$-Grenzproze ${ }^{17}$ oder vom Standpunkt der verallgemeinerten Feldtheorie eine spezielle Wahl der das Potential erzeugenden Funktion $f(\sigma)^{18}$. Jetzt treten in der Hamilton-Funktion keine expliziten Unendlichkeiten mehr auf. Die Schrödinger-Gleichung

$$
-\frac{\hbar}{i} \frac{\partial \psi}{\partial t}=H \psi
$$

hat also in der gewöhnlichen Quantenelektrodynamik in Strenge bestenfalls erst einen Sinn bei Benützung des $\lambda$-Prozesses oder anderer Grenzprozesse. Auch bei der Lösung der Schrödinger-Gleichung mit Grenzprozeß treten wieder Divergenzen auf, die nach der Diskussion im Abschnitt 2 ihren Grund in den Schwankungen des quantisierten Feldes haben. Bei dieser Lösung ist jedoch das Störungsverfahren benutzt, so daß die transversale Selbstenergie im Gegensatz zur statischen nur in einer Näherung bekannt ist. Wie das oben angeführte Beispiel zeigt, kann daraus noch nicht mit Sicherheit auf die Divergenz des exakten Ausdrucks geschlossen werden. Hier ist es jedoch möglich, an eine nicht sehr bekannte Arbeit von Gust a f s o n ${ }^{13}$ anzuknüpfen. Dort wird die Schrödinger-Gleichung in einer (2.3) entsprechenden Form als unendliches Differentialgleichungssystem benutzt (siehe $\mathrm{II}^{4}$, Abschnitt 1). Setzt man dann

$$
a_{m}(t) e^{-i E_{m} t / \hbar}=z_{m} e^{-i r t / \hbar},
$$

so erhält man das folgende homogene Gleichungssystem

$$
\left(r-E_{m}\right) z_{m}=\sum_{n} H_{m n} z_{n},
$$

das bekanntlich nur dann nicht triviale Lösungen besitzt, wenn $r$ eine Wurzel der Säkulardeterminante $D_{i k}$

$$
D_{i k}=\left|H_{i k}-\left(r-E_{k}\right) \delta_{i k}\right|=0
$$

darstellt. Wie sich aus den das obige Vorgehen rechtfertigenden physikalischen Gründen leicht ergibt, bilden die Wurzeln $r_{v}$, der Säkulardeterminante gerade die Energieeigenwerte des Gesamtsystems. Für ein ruhendes Elektron, das nur in Wechselwirkung mit

17 P. A. M. D i r a c, Proc. Roy. Soc. [London], Ser. A, 180, 1 [1942]; Com. Dublin Inst. Adv. Studies, Ser. A, Nr. 1 [1943]. seinem Strahlungsfeld steht, gilt also

$$
r_{0}=\mu+\Delta E_{0},
$$

wobei $\Delta E_{0}$ die exakte transversale Selbstenergie des Elektrons darstellt. Gustafson berechnete nun $r_{0}$ in der Quantenelektrodynamik ohne Auffüllung der negativen Energieniveaus bei Wechselwirkung des Elektrons mit $n$ Oszillatoren des Strahlungsfeldes, um $\mathrm{zu}$ untersuchen, ob durch das oft als Notbehelf verwendete sogenannte Abschneiden (d. h. man integriert z. B. in (1. 1) nur von 0 bis zu einem größten Impuls $K)$ überhaupt die Endlichkeit des gesamten Selbstenergieausdrucks gewährleistet wird. Die Glieder der aus der Störungstheorie folgenden Reihenentwicklung sind dann zwar alle endlich, doch ist damit noch nichts über die exakte Selbstenergie bei Benützung eines Abschneideverfahrens gesagt. Dem Abschneideverfahren entspricht nun in der Methode von Gustafson die Beschränkung, daß das Elektron nur mit den im Impulsintervall von 0 bis $K$ liegenden Oszillatoren in Wechselwirkung treten kann. Da wegen der Einschließung in ein Periodizitätsvolumen alle Energien und Impulse nur diskrete Werte annehmen können, ist diese Oszillatorenzahl endlich (etwa gleich $n$ ). Gustafson fand dabei, daß Konvergenz des Selbstenergieausdrucks nur bei Wechselwirkung mit einer Oszillatorenanzahl unterhalb einer festen endlichen Schranke im Einheitsvolumen stattfindet. Für die exakte transversale Selbstenergie, wie sie sich ohne Abschneiden ergibt, hat Gustafson daraus keine weiteren Schlüsse gezogen. Doch können wir jetzt unmittelbar daraus folgern, daß wenn wir $n$ so groß wählen, daß es jedenfalls über der von Gustafson gefundenen festen endlichen Schranke liegt, der exakte Selbstenergieausdruck divergent ist. Benützen wir nun gar kein Abschneideverfahren, so ist die Zahl der Oszillatoren des Strahlungsfeldes, mit denen das Elektron in Wechselwirkung treten kann, unendlich groß, also immer über der Schranke von Gustafson gelegen. Damit ist der exakte Ausdruck für die transversale Selbstenergie in der gewöhnlichen Quantenelektrodynamik als divergent erwiesen, d. h. die zugehörige Schrödinger-Gleichung besitzt auch mit Benützung des $\lambda$-Prozesses oder anderer Grenzprozesse, die die statische Selbstenergie in invarianter Weise eliminieren, zu endlichen Energieeigenwerten keine exakte Lösung.

Dieser Schluß läßt sich jedoch nicht unmittelbar in

18 F. B o p p, Ann. Physik (5) 42, 573 [1942] \& 5, S. 595 oder auch Z. Naturforschg. 3a, 564 [1948], Einleitung, insbesondere Gl. (4). 
die Positrontheorie übertragen. Die Wechselwirkung mit den unendlich vielen Vakuumelektronen negativer Energie verändert bereits die elektrostatische Selbstenergie des Elektrons in solcher Weise, daß sie ebenso wie die transversale Selbstenergie in der Positrontheorie logarithmisch divergiert und ebenfalls wie diese nur in störungstheoretischen Näherungen berechnet werden kann. Versucht man nun eine exakte Behandlung der Selbstenergie in der Positrontheorie durchzuführen, so trifft man auf die fundamentale Schwierigkeit, daß das exakte Problem gar nicht vollkommen definiert ist. Schon in höheren störungstheoretischen Näherungen treten bekanntlich die Mängel an Eindeutigkeit hervor ${ }^{19}$. Um also diesem Fragenkreis des Verhaltens der exakten Selbstenergie näherzutreten, müßte man vorher erst eine

19 Erst in der letzten Zeit hat F. J. D y s o n, Physic. Rev. (2) 75, 1736 [1949], im Zusammenhang mit der Tomonaga-Schwingerschen Masse-Ladungsrenormierung genaue Vorschriften für die Behandlung der höheren störungstheoretischen Näherungen gegeben. definiertere Positrontheorie schaffen. Mit der bisherigen Formulierung kann man ganz verschiedenartige „exakte“ Ergebnisse erhalten ${ }^{20}$. Wir mußten uns deshalb bezüglich der exakten Behandlung auf eine genauere Diskussion der Quantenelektrodynamik ohne Löchertheorie beschränken.

Die heutigen Schwierigkeiten in der Quantenelektrodynamik sind also nicht durch eine unpassende mathematische Methode verursacht, sondern in den Grundlagen der Theorie enthalten. Sie sind teilweise klassischen und teilweise quantentheoretischen Ursprungs. Die klassischen haben ihren Grund in der unzutreffenden Formulierung der Eigenkräfte (genauer der Trägheitskräfte) von punktförmigen Teilchen, die quantentheoretischen in den Schwankungen der quantisierten Felder.

Auf die weiteren sich daraus ergebenden Fragen soll in II eingegangen werden.

20 Zusatz b. d. Korr.: Dies gilt auch bei Benutzung der kovarianten Formulierung von $\mathrm{T}$ o $\mathrm{m}$ o $\mathrm{n}$ a g a.

\title{
Der Tritiumgehalt der Atmosphäre
}

\author{
Von V. Faltings und P. Harteck \\ Aus dem Physikal.-chem. Institut der Universität Hamburg \\ (Z. Naturforschg. 5 a, 438-439 [1950]; eingegangen am 2. August 1950)
}

\begin{abstract}
Tritium konnte im freien Wasserstoff der Atmosphäre, welcher zu Wasser verbrannt worden war und elektrolytisch eingeengt wurde, an seiner $\beta$-Aktivität nachgewiesen werden. In $10 \mathrm{~cm}^{3}$ Luft ist etwa 1 Tritium-Atom vorhanden, in der ganzen Atmosphäre $1 \mathrm{Mol}$.
\end{abstract}

$D_{s}$ er Entdeckung des Tritiums in der Atmosphäre gehen eine Reihe von Arbeiten voraus, die hier zunächst kurz geschildert werden mögen. H. J e n s e $n$ und der eine von uns hatten gezeigt ${ }^{1}$, daß in den höchsten Schichten der Atmosphäre der Wasserdampf durch das Ultraviolett der Sonne zerspalten wird, die H-Atome das Schwerefeld der Erde verlassen und der Sauerstoff zurückbleibt, wodurch die dauernden Sauerstoffverluste durch Oxydation der Gesteine über die geologischen Epochen ausbalanciert werden. In einer Zuschrift an die „Naturwissenschaften " 2 haben H. S u es s und der eine von uns über Experimente berichtet, in denen gezeigt wurde, daß der elementare Wasserstoff, welcher in einer Konzentration von $5 \cdot 10^{-7} \mathrm{~mol} / l$ in der normalen Luft enthalten ist, in seiner isotopischenZusammensetzung vom Normalen abweicht, da das Verhältnis D:H=1:4500

1 H. J ens en, Z. Naturforschg. 3a, 591 [1949]. gefunden wurde gegenüber der normalen Zusammensetzung von 1:6000. Wasserstoff, der aus Fäulnisprozessen usw. an der Erdoberfläche entsteht, kann nur einen geringeren D-Gehalt als normaler Wasserstoff besitzen, da im chemischen Gleichgewicht bei Zimmertemperatur mehr als 3-mal soviel D im Wasser als im Wasserstoff enthalten ist. Trotzdem ist der D-Gehalt des aus dem Wasserstoff der Luft gewonnenen Wassers höher als der des normalen Wassers. Dies kann offenbar nur so verstanden werden, daß der Effekt des bevorzugten Wegdiffundierens von H-Atomen aus dem Schwerefeld der Erde und die dadurch hervorgerufene D-Konzentrationserhöhung das soeben erwähnte Moment überkompensiert. Dieses Ergebnis zeigt aber auch, daß der in den höchsten Schichten der Atmosphäre zu HD rekombinierte Wasserstoff nur teilweise in

2 H. S u e s s, Naturwiss. 36, 218 [1949]. 